\title{
BMC Obesity reviewer acknowledgement 2015
}

Clare Partridge

\section{Contributing reviewers}

The editors of BMC Obesity would like to thank all our reviewers who have contributed to the journal in Volume 2 (2015).

Gavin Abbott

Australia

Asnawi Abdullah

Indonesia

Jesmin Akter

Bangladesh

AMAAP Alagiyawanna

Sri Lanka

Navoda Atapattu

Sri Lanka

Tom Barber

UK

Fiona Kate Barlow

Australia

Monica Baskin

USA

Sanjay Basu

USA

Grzegorz Bazylak

Poland

Alison Beauchamp

Australia

Rebecca Beeken

UK

Britni Belcher

USA
Colin Bell

Australia

Ragnar Bjarnason

Iceland

Catriona Bonfiglioli

Australia

Paula Brauer

Canada

Andrew Breck

USA

Anna-Kristin Brettschneider

Germany

Adrian Brown

UK

Maria Bryant

UK

Robert Caesar

Sweden

\section{Adrian Cameron}

Australia

Dexter Canoy

UK

Gianluca Castelnuovo

Italy

Mu Chen

USA

\author{
Mansi Chopra \\ India \\ Paul Collings \\ UK
}

Amanda Daley

UK

Hélène Delisle

Canada

Jacopo Desiderio

Italy

Hossam Draz

Saudi Arabia

John Dube

USA

Kate Ellacott

UK

Wayne English

USA

Eshan Fernando

Canada

Rebecca Firestone

USA

Helena Fonseca

Portugal

Rosanne Freak-Poli

Australia

Correspondence: clare.partridge@biomedcentral.com

BioMed Central, Floor 6, 236 Gray's Inn Road, London WC1X 8HB, UK 
L M Funk

USA

Frances Garden

Australia

Jewel Gausman

USA

Gilles Gouspillou

Canada

Josephine Gwynn

Australia

Nyssa Hadgraft

Australia

Mark Harris

Australia

Hongbo He

China

David Heber

USA

Peter Henneman

Netherlands

Erica Hinckson

New Zealand

Bruce Hollis

USA

Katy Horner

Ireland

Golam Hossain

Bangladesh

Haijing Huang

USA

Fumiaki Imamura

UK

Lindsay Jaacks

USA

Chandra Jackson

USA

G S Jakobsen

Norway

Randi Jepsen

Denmark

Rodney Joseph

USA

Nishan Kalupahana

Sri Lanka
Anne Kavanagh

Australia

Isurujith Kongalaliyanage

Sri Lanka

Vivica Kraak

USA

Yan Lam

Australia

Donatella Lanari

Italy

Rachel Laws

Australia

Dayna Lee-Baggley

Canada

Jimmy Chun Yu Louie

Australia

Julie Lumeng

USA

Brigid Lynch

Australia

Claire Madigan

Australia

Kristen Malecki

USA

Lucia Martinez De La Escalera Clapp

USA

Paolo Marzullo

Italy

Stephanie Mazzucca

USA

Philip McTernan

UK

Alexander Miras

UK

Jason Nagata

USA

Christine Olson

USA

Rocco Paluch

USA

Dimitris Papamargaritis

UK

Guillaume Pare

Canada
Ashan Pathirana

Sri Lanka

Christine Peat

USA

Melanie Pescud

Australia

Helena Piccinini-Vallis

Canada

Milan Piya

UK

Virginia Quick

USA

Elizabeth Reifsnider

USA

Jinnie Rhee

USA

Wendy Robertson

UK

Liane Roe

USA

Bashiru Imoro Ibn Saeed

Ghana

Francesca Seta

USA

Florian Seyfried

Germany

Carrie Sheets

USA

Saad Siddiqui

Oman

Jamie Sims

UK

Debbie Smith

UK

Nicola Spurrier

Australia

Claire Stocker

UK

Claudia Strugnell

Australia

Nicole Stupka

Australia

Aaron Sussell

USA 
Abd Tahrani

UK

Lisa Te Morenga

New Zealand

Simon Thornley

New Zealand

Qingchun Tong

USA

Léonie Uijtdewilligen

Singapore

Cherian Varghese

Switzerland

Madhusudhan Varma

UK
Anthony Viera

USA

Ramon Vilallonga

Spain

Tommy Visscher

Netherlands

Indu Waidyatilaka

Sri Lanka

Connie Weaver

USA

Brandy Wicklow

Canada

Pujitha Wickramasinghe

Sri Lanka
Cameron Willis

Canada

Tsedeke Wolde

Ethiopia

Evelyn Wong

Australia

Yao Yao

USA

Alfred Yawson

Ghana

Changzheng Yuan

USA

Michael Zulyniak

Canada 